\title{
Damage Diagnosis of Bolt Loosening via Vector Autoregressive - Support Vector Machines
}

Mahmut Pekedis

Ege University, Department of Mechanical Engineering, Izmir, Turkey

A B S T R AC T
evelopments in engineering techniques have concentrated on how to build better solu-
tions for engineering structures in order to main the integrity and to reduce the costs
in operations. Since the last two decades, advances in computational power have allowed
machine learning algorithms to be applied as a powerful tool in anomaly detection problems,
classification as well as in regression analysis. The objective of this study is to detect the
damage using the vector auto regression model (VAR) coupled with support vector machines
(SVM). A base excited three storey manufactured from an aluminium is investigated in a
lab medium for various structural states. Accelerometers are fastened to the each corner of
structure's floor to collect time series data. Damage simulation scenarios in structure are per-
formed by releasing the bolt load which cause the nonlinear effects. Once the sensors' meas-
urements are collected for each state and organized to represent the appropriate scenario's
label, they are processed in VAR model to obtain feature vectors such as residuals and VAR
parameters. Then, SVM with optimal kernels are implemented on those features to classify
and locate the damage. The results demonstrate that the VAR residuals shows a significant
performance over VAR parameters once they are used as features in SVM technique. Moreo-
ver, it is also found that detection performance rises as the number of damage increases.

\author{
Article History: \\ Received: 2019/12/10 \\ Accepted: 2020/09/08 \\ Online: $2020 / 09 / 30$ \\ Correspondence to: Mahmut Pekedis, \\ Deparment of Mechanical Engineering, \\ Ege University, İmir, Turkey \\ Tel: +902323114970 \\ Fax: +902323888562 \\ E-Mail:mahmut.pekedis@ege.edu.tr
}

\section{Keywords:}

Structural health monitoring, Pattern recognition, Machine learning, Damage diagnosis, Vector autoregressive - Support vector machines

\section{INTRODUCTION}

$\mathrm{T}$ he engineering systems are the potential damage accumulated structures at environmental and operational conditions. The most research conducted in structural health monitoring (SHM) has focused on ways of detecting the damages in these structures at the earlier possible time. The ability of monitoring accurately the all parts of the structure is crucial to improve both the reliability and safety. During the last two decades, the structural monitoring techniques have been focused to diagnose the damage by equipping the structure with various types of sensor. One such a monitoring procedure is vibration based damage identification which is based upon the changes in dynamic response such as crack, stiffness reduction or loosed connection that alter the response of that structure [1-4]. There has been a much research on this field such as Doebling et al. presented a review for the vibration based damage detection techniques [5]. The model based SHM approach is generally implemented by building a physics based model for the system. Once the model of the system is established based on a physical description, it is updated on measurements obtained from sensors that attached to structure. Although the much of vibration based SHM studies were concentrated on model based SHM, Farrar et al., have presented statistical pattern recognition paradigm for diagnosing the damage using direct data of vibrations [6]. This paradigm includes of four parts: (a) operational evaluation, (b) data acquisition, (c) feature extraction and reduction, (d) statistical model development. Recent advancements regarding of this technique is demonstrated in detail by Farrar and his coworker [7].

The algorithms mostly used in statistical model development can be categorized as, (1) classification [7-8], (2) regression [9] and (3) outlier detection [10]. The suitable technique to be selected is based on the capability of performing unsupervised or supervised learning. Supervised learning depicts that, the data of undamaged and damaged conditions are available. It is mostly used in group classification. However, unsupervised learning refers the structural state where the data are available 
only for the healthy state of the system. This procedure is performed in SHM techniques to fit a time series predictive model. Once the model is set, the anomalies can be checked by using the observations. In other words, the residuals are used as a sensitive features for the damages. Then, the changes in residuals are considered to be caused due to damage in structure. Typically, linear based autoregressive (AR) model [11-12] and non-linear based autoregressive SVM [9] are employed on time series to compute the damage sensitive feature. After the model has been established, the residual parameter which reflects the damage indicator has been interpreted with procedures such as sequential probability test [13] and sliding window [14]. The majority of the studies reported above have used auto regressive model (AR) model as damage sensitivity vector [9-12]. However, AR model does not include the relation between multiple time series. On the other hand, vector auto regression (VAR) which is a generalized form of univariate AR Model captures the interdependencies between multivariate time series. Here, we propose a VAR (Vector Autoregressive) model coupled with SVM (Support Vector Machines) technique to diagnose and classify the damage using an array of sensors' measurements. The study is investigated on a base excited three storey in a lab condition. The damage is performed by releasing the bolt load which causes a nonlinear effect on the system response. The measuring data of the sources that caused from variability such as temperature, humidity or external noises are not essential herein this technique.

The layout of this study is as follow. First, the basic theory of VAR - SVM framework is briefly described. Next, experimental setup of the test structure is demonstrated. Then, the results obtained from the approach is presented in detail. Finally, the diagnosing performance is summarized and discussed.

\section{VECTOR AUTO REGRESSIVE - SUPPORT VECTOR MACHINE MODEL}

Consider $\left\{x_{1}, x_{2}, \ldots, x_{\mathrm{T}}\right\}$ are zero mean time multivariate time series collected from SHM networks. Each $x_{t}$ sensor $\left\{x_{t 1}, x_{t 2}, \ldots, x_{t m}\right\}$ is the vector observations, $\mathrm{m}$ is the total number of sensor, $\mathrm{T}$ is time points and $\mathrm{x}_{\mathrm{ti}}$ is the observation of ith sensor at time t. A VAR model is described as

$$
x_{t}=\sum_{i=1}^{p} \varphi_{i} x_{t-i}+\varepsilon_{t}
$$

where $\mathrm{p}$ is the order of the model and $\varepsilon_{t}=\left\{\varepsilon_{t 1}, \varepsilon_{t 2}, \ldots, \varepsilon_{t m}\right\}, \varepsilon_{t} \in R$ are the residuals having normal distribution. The $\Phi_{1}, \Phi_{2}, \ldots, \Phi_{p}$ parameters can be estimated by maximum likelihood method [15-16]. The optimum order of the model is predicted by Akaike [17] and Bayesian [18] information criterions as following equations.

$$
\begin{aligned}
& A I C(p)=-2 \ln L(p)+2 k \\
& B I C(p)=-2 \ln L(p)+k \ln T
\end{aligned}
$$

where $\mathrm{k}$ is the total parameter number of the model, $\mathrm{T}$ is the total observation points and $\mathrm{L}(\mathrm{p})$ is likelihood of the model. These criterions provide details about how the predicted parameters fit into the model. The model parameters and residuals are sensitive to varieties for structures in environmental and operational conditions. Each of these feature computed for various case is labeled with a structural case, then processed in support vector machines (SVM) to diagnose the damage. Briefly, SVM is a statistical technique which seeks the separate of classes [19]. Initially, the theory of SVM has been discussed for separation of two class data, then extended to separate of multi classes data [20].

Consider the training dataset contains $N$ input vectors such that $\mathrm{x}_{1}, \mathrm{x}_{2} \ldots \mathrm{x}_{\mathrm{N}}$, and target values $t 1, \ldots t_{N}$, whe$\operatorname{ret}_{N} \in\{-1,1\}$, the new data are classified in $D\left(\underline{x}_{k}\right)$.

The linear separation situation is

$$
\begin{aligned}
& \underline{x}_{k} \in C_{1} \Rightarrow D\left(\underline{x}_{k}\right)=w \underline{x}_{k}+b \geq 1 \\
& \underline{x}_{k} \in C_{2} \Rightarrow D\left(\underline{x}_{k}\right)=w \underline{x}_{k}+b \leq-1
\end{aligned}
$$

where $C_{1}, C_{2}$ refer the class label, $w$ is the weight vector and $\mathrm{b}$ is bias parameter [21].

Equation (4) could be written in Euclidean space as following

$$
D\left(\underline{x}_{k}\right)=y_{k}\left\langle\underline{w}, \underline{x}_{k}\right\rangle \geq 1
$$

where $y_{k}$ is a class label.

The distance of each data to the separated hyperplane is defined as

$$
y_{k} \frac{D\left(\underline{x}_{k}\right)}{\|\underline{w}\|} \geq \tau
$$

where $\tau$ is the distance between two hyper planes. The parameterization of the hyper plane is unreasonable. It could be adjusted as 


$$
\|\underline{w}\| \tau=1
$$

The maximizing the margin can be conducted by minimizing the $\|w\|$. Then, the optimization problem simply requires that, $\min \left[\frac{1}{2}\|w\|^{2}\right]$ is to be maximized. By minimizing the $\|w\|$ and subjected to constraints defined in equation (5), the objective function is defined as

$$
Q(\underline{w})=\frac{1}{2}\|\underline{w}\|^{2}-\sum_{i=1}^{N} \alpha_{i} y_{i}\left(\left\langle\underline{w}, \underline{x}_{i}\right\rangle-1\right)
$$

where $\alpha_{i}$ are the Lagrange multipliers. It is noted that, the minus sign of the Lagrange multiplier term is due to minimization with respect to $w$. The parameters of $w$ could be written in terms of $\alpha_{i}$ by performing Kuhn Tucker conditions. Then, the dual formulation will be as

$$
\frac{\partial Q}{\partial \underline{w}}=0 \quad \Rightarrow \underline{w}=\sum_{i=1}^{N} \alpha_{i} y_{i} \underline{x}_{i}
$$

The Lagrange multipliers $\alpha$ i only can be nonzero if the constraints hold with

$$
\alpha_{i} y_{i}\left(\left\langle\underline{w}, \underline{x}_{i}\right\rangle-1\right)=0
$$

The data points xi observed at margin limits are named support vectors. The formulation of dual could be maximized with respect to $\alpha$ i by substituting equation (10) into (8) such that

$$
Q(\underline{w})=\sum_{i=1}^{N} \alpha_{i}-\frac{1}{2} \sum_{i=1}^{N} \sum_{j=1}^{N} \alpha_{i} \alpha_{j} y_{i} y_{j}\left\langle\underline{x}_{i}, \underline{x}_{j}\right\rangle
$$

subjected to the following constraints

$$
\sum_{i=1}^{N} y_{i} \alpha_{i}=0 \quad \alpha_{\mathrm{i}} \geq 0, i=1,2,3, \ldots, N
$$

After the optimal parameter is determined by gradient descent or quadratic programming, the optimal separating hyperplane given in equation (9) could be written as the following equation

$$
D(\underline{x})=\sum_{i=1}^{N} \alpha_{i} y_{i}\left\langle\underline{x}_{i}, \underline{x}\right\rangle
$$

This statement provides that the data are separable linearly. If they are not separable linearly, $\xi_{i}$ slack variables are added to the non separable data as

$$
D\left(\underline{x}_{i}\right)=y_{i}\left\langle\underline{w}, \underline{x}_{i}\right\rangle \geq 1-\xi_{i}
$$

where ${ }^{\xi_{i}}$ assess the non-separability. This can be solved by adding the penalty form $\sum_{i=1}^{N} \xi_{i}^{p}$ to the objective function.

The essential objective function is defined as

$Q(\underline{w})=\frac{1}{2}\|\underline{w}\|^{2}+C \sum_{i=1}^{N} \xi_{i}-\sum_{i=1}^{N} \alpha_{i} y_{i}\left(\left\langle\underline{w}, \underline{x}_{i}\right\rangle-1+\xi_{i}\right)-\sum_{i=1}^{N} \mu_{i} \xi_{i}$

and subject to the following constraints

$$
\sum_{i=1}^{N} y_{i} \alpha_{i}=0 \quad C \geq \alpha_{i} \geq 0, \quad i=1,2,3 \ldots \ldots . N
$$

where the $C$ value arranges the complexity for the model. The procedure is applied on nonlinearly separable datasets by asserting them in high dimensions $[20,22]$.

The optimization includes the following objective function in high dimensional space

$$
Q(\underline{w})=\sum_{i=1}^{N} \alpha_{i}-\frac{1}{2} \sum_{i=1}^{N} \sum_{j=1}^{N} \alpha_{i} \alpha_{j} y_{i} y_{j}\left\langle\left\langle\varphi\left(\underline{x}_{i}\right), \varphi\left(\underline{x}_{j}\right)\right\rangle\right\rangle
$$

where $\langle\langle\rangle$,$\rangle depicts the inner product on the feature$ space. The solution of CPU time increases as the feature space increases.

The resulting nonlinear discriminant function for two label classes is given by

$$
D\left(\underline{x}_{i}\right)=\sum_{i=1}^{N} \alpha_{i} y_{i} H\left(\underline{x}_{i}, \underline{x}\right)
$$

where $H\left(\underline{x}_{i}, \underline{x}_{j}\right)$ is assessed for input space 
$\left\langle\left\langle\varphi\left(\underline{x}_{i}\right), \varphi\left(\underline{x}_{j}\right)\right\rangle\right\rangle$. if $D\left(\underline{x}_{i}\right) \geq 0 ; \mathrm{x}$ is designated on the class

label 1; else, $\mathrm{x}$ is designated on the class label 2. Most com-

monly used kernel functions $\varphi\left(\underline{x}_{i}, \underline{x}_{j}\right)$ in SVM are polyno-

mial, normalized, polynomial, radial based and sigmoid. These functions are described by Bishop in detail [20]. It is noted that, if the parameters of the kernels are not optimized, low classifications performance could be occurred due the separation problems of hyperplanes. The VAR-SVM model is constructed using MATLAB econometrics, Statistics and Machine Learning Toolbox. The regularization $C$ in SVM is implemented via the box-constraint parameter with a value of 1 . It is noted that as this value increases more points are allowed in the margin. Hence, this regularization relaxes this constraint. The parameters processed in VAR are also standardized by centering and dividing them by their standard deviations.

The methodology used in VAR-SVM model is as follows:

a. Initially, the VAR model is established for the raw sensor measurements. The optimum model order $p$ is determined at minimized values of $\mathrm{AIC}(\mathrm{p})$ or BIC(p) function.

b. The VAR model is re-established with an optimised p order. Then, the VAR parameters and residual vectors are used as features in SVM.

c. Once the features data are obtained from VAR model for various cases, they are partitioned into two groups. The 1st group is the training dataset. This training group is then separated into more two groups. One training group is used to develop the SVM model and the other is determine the optimal kernel parameters.

d. The 2nd group is testing dataset. This group is used to determine the performance of the model that is created in the first group. Once the kernel parameters are optimized in first group, the diagnosing performance of SVM is assessed on testing dataset.

\section{EXPERIMENTAL SETUP}

Three storey structure made of aluminum frames is manufactured as a test platform (Fig. 1). The structure can slides on rails that allow movement in lateral direction only and its ground storey has been excited with an electromagnetic shaker vibrating at band limited random frequencies $(15-150 \mathrm{~Hz})$. In order to prevent the system to move away during excitation, a tensional spring is placed between the lower surface of structure and the base plate. It is noted that the stretch magnitude of this spring can be controlled with a bakelite screw. The connections of columns, frames and lower base are carried out by bolts. The loosen bolt during excitation yields in a non-linear behavior that represents a damages. In other words, exciting the system while no joints loosened presents a linear response of structure, while loosed joints result in a nonlinear dynamic response.

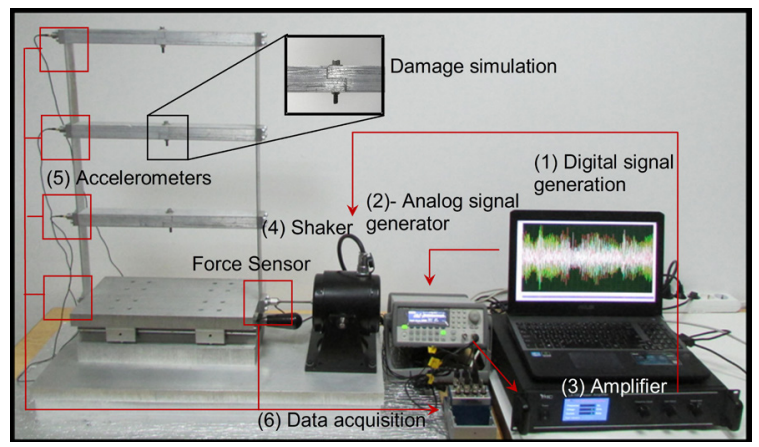

Figure 1. Experimental setup

The structure which located on base plate is excited by a YMC, MS-50 type electrodynamics shaker. This shaker is amplified with a YMC, LA-100 instrument. This amplifier is embedded with a signal generator that can only produce sine signals. However, the real world engineering systems are exposed to environmental and operational uncertainties. In order to simulate this variability, the system is excited with band limited signals generated by Agilent 33210A.The procedure steps that we deployed are numbered in Fig 1, and these are briefly as follows. Initially, band limited digital random signals (1) are transferred to Agilent 33210A on TCP/ IP connection to generate analog signals (2). Then, these analog signals are amplified in YMC, LA-100 (3). Finally, the amplified band limited analog signals are transferred to the shaker to excite the structure (4). The base plate slides on rails and the entire system (three storey, shaker) is placed at a foam to minimize external sources of unmeasured excitation. Four YMC piezo accelerometers have a sensitivity of $9.39 \mathrm{mV} / \mathrm{g}$ are attached on the corner of each storey (5).

The shaker is centered at the edge of base plate. Moreover, the structure and accelerometers are placed on the same axes line of base plate's axis to reduce the torsional effects during excitation. The measurements are collected with NI 9234 (6). The force sensor at base floor and accelerometers located at 1st, 2nd and 3rd floor are connected to channel 1, 2, 3, 4 and 5 of the device, respectively. Then, these measurements are processes with Labview software. Totally, 8298 data points are acquired with a sampling rate of 1765.53 $\mathrm{Hz}$ for each case. The structure is excited at band limited random frequencies at $15-150 \mathrm{~Hz}$. Note that, these levels are chosen to avoid rigid motion caused by 1st mode, and these values are slightly above the first mode. The excitation level of amplifier is set to be as 3.4 V RMS (root mean square error). The datasets collected for these cases are categorized in accordance for bolt looose variation as given in Table 1. Here, while the case 1 represents the healthy condition, the rest 7 cases shows the damaged states. Note that, each datasets given in Table 1 consist of 6 dimensions where 
the 1st is the time (s), 2nd is force $(\mathrm{N})$, and the rests are accelerations (g). Since the system consist of 3 horizontal frames including loosing bolts, eight different damage cases combinations $2^{3}=8$ could be investigated. We note that, there is not a general rule about how many repeated datasets should be collected for each test. However we know that as the number of case is increased higher datasets are needed to be collected. Initially, in order to determine to each algorithm' classification sensitivity the investigations have been performed for the 4 cases listed in Group I (Table 1). Then, the algorithm which reflect the highest accuracy has been implemented for all cases given in Group I and II.

Table 1. Structural state cases

\begin{tabular}{ccccc}
\hline & \multirow{2}{*}{$\begin{array}{c}\text { Case } \\
\text { no }\end{array}$} & \multicolumn{4}{c}{ Bolt loose option } \\
\cline { 3 - 5 } & 1 & 1st floor & 2nd floor & 3rdfloor \\
\hline Group I & 2 & - & - & - \\
& 3 & - & $\bullet$ & $\bullet$ \\
& 4 & $\bullet$ & $\bullet$ & $\bullet$ \\
Group II & 5 & $\bullet$ & - & - \\
& 7 & $\bullet$ & $\bullet$ & $\bullet$ \\
& & & & $\bullet$
\end{tabular}

- " represents the bolted joint is loosed, while "-" shows the bolt is not loosed

\section{RESULTS}

In this study, machine learning based active structural health monitoring techniques have been implemented on a base excited three storey structure to diagnose the bolt loosening damage. In order to include the effects of environmental and operational varieties, this system is excited by a band limited random vibrations. The raw measurements includes time $(\mathrm{s})$, force $(\mathrm{N})$ and accelerations (g) for a typical dataset are represented in Fig 2. The

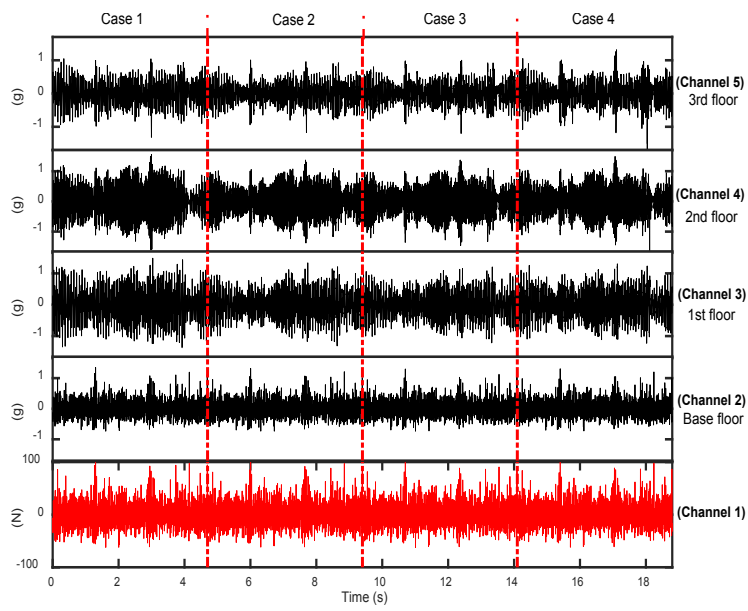

Figure 2. Typical raw data measurements of the 1st dataset sensor measurements located at base floor (Channel 1 and 2) show more random distributions due to excitation.

For the duplicated measurements, uncertainties may arise at different stages of a measurement process. In order to evaluate these uncertainties, the measurements have been evaluated using robust statistical techniques such as cumulative density function, Z-score and analysis of variance (ANOVA). For instance, the cumulative density function distribution of the discrete data obtained for the 4 repeated tests at Channel 1 and 5 are represented in Fig 3 and Fig 4,

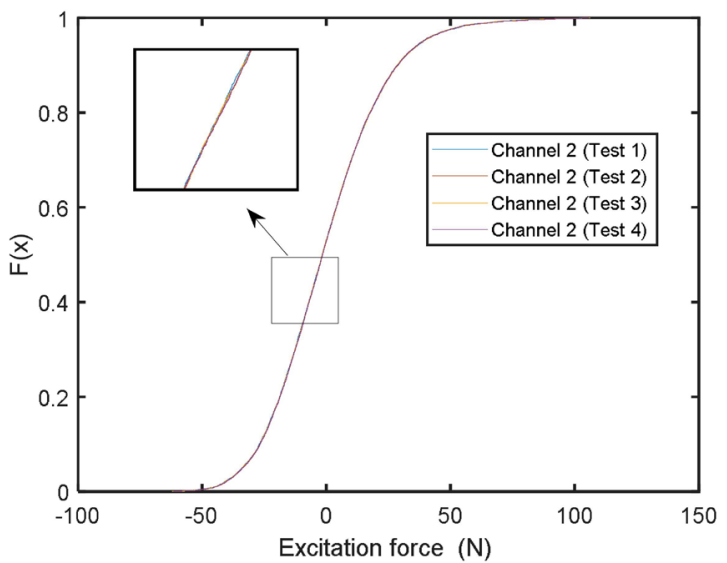

Figure 3. Cumulative density functions for the repeated raw force measurements [case 1].

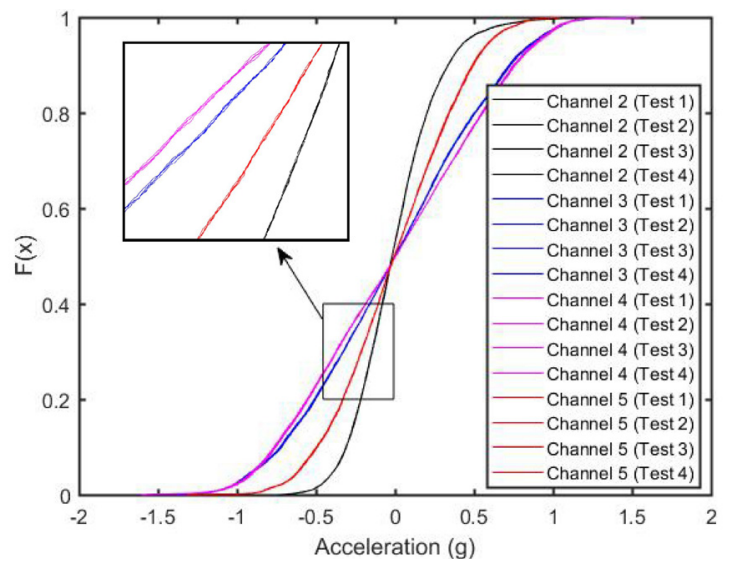

Figure 4. Cumulative density functions for the repeated raw acceleration measurements [case 1].

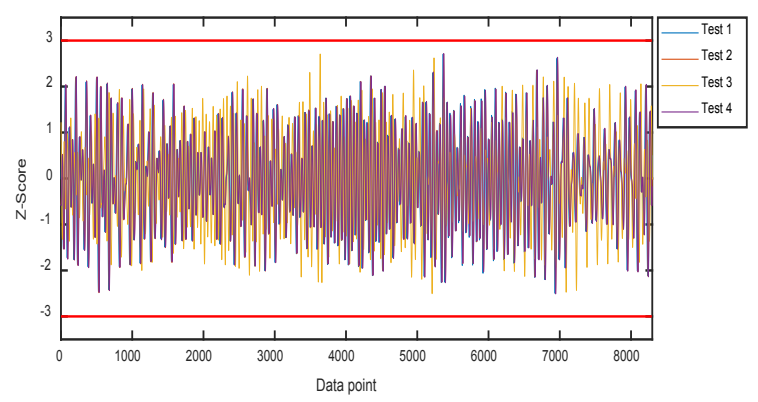

Figure 5. Z-scores of the raw measurements obtained for repeated raw acceleration measurements [case 1, Channel 4]. 
respectively. The duplicated measurements converge each other which may show the uncertainty due to the DAQ device is low.

Next, uncertainty is also evaluated using Z-scores based on robust statistics. It is widely implemented for the processing of experimental data obtained from inter laboratory comparisons [23-24]. Here, Z-score gives a measurement of how far a result is from the assigned value, and also gives a score to each result relative to the other results in the group. The measurement uncertainty can be estimated from Z-score and a value less than 3 is accepted an appropriate from the data group. In other words, $|\mathrm{Z}| \leq 3.0$ indicates a satisfactory performance [23]. For instance, Z-scores of the duplicated measurements are in the range of -3 and 3 (Fig 5). Moreover, these signals do not include outliers.

We finally have evaluated the raw measurement uncertainty using box plots. Typically, the box plot is a standardized method of representing the distribution of samples corresponding to features using boxes and whiskers. The boxes show the inter quartile range of the data and the whiskers refer a multiple of the first and third quartiles of the variable. Any data that laid at the outside of this limit is considered as outliers. Box plots based on Z-scores for the raw repeated measurements for various channels are given in Fig 6. Note that each test data in each channel consist of 8298 data points. It can be seen that while the channel 2 and 5 include outliers, channel 3 and 4 do not. For instance, test data 1, 2, 3 and 4 from the channel 1 include 162, 154, 149 and 159 outliers. Additionally, data from channel 4 covers 10, 11, 11, 11 outliers for test 1,2,3 and 4 respectively. We expect that the reason of higher outliers encountered in Channel 2 may occurred due the this sensor is laid at the excitation floor which include random distribution.
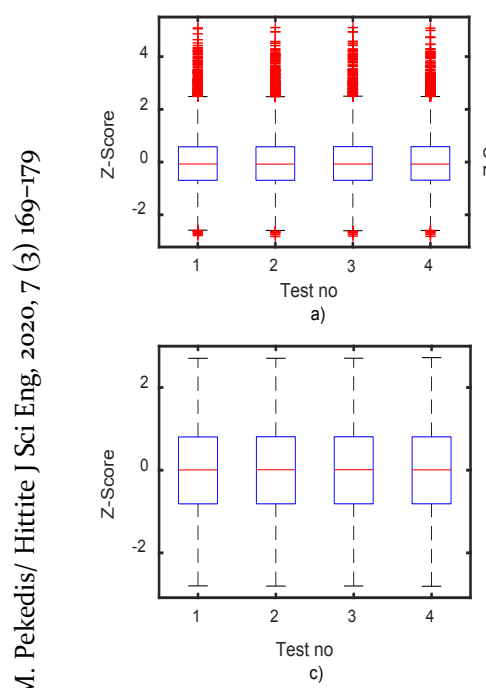

c)

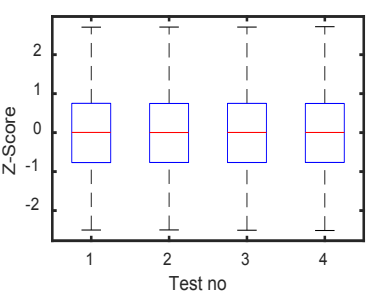

b)

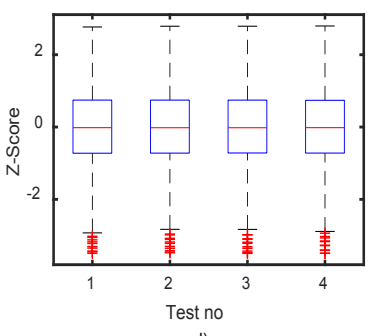

d)
Figure 6. Box plots of the Z-scores for the raw repeated measurements[case 1]. a) Channel 2, b) Channel 3, c) Channel 4, d) Channel 5 a)

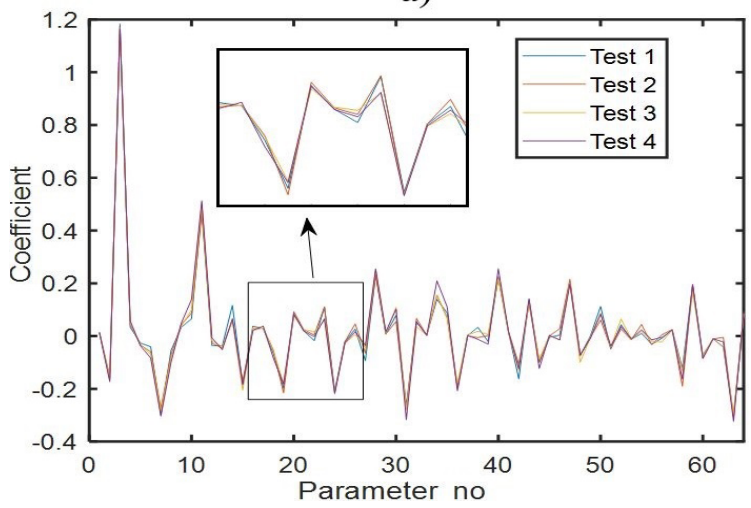

b)

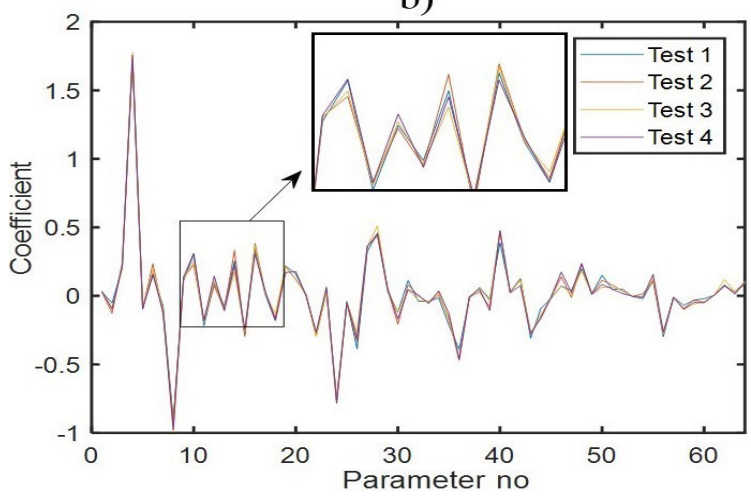

Figure 7. VAR(16) parameters distribution for various repeated tests [case 1]. a) Channel 4, b) Channel 5

Once the datasets are collected and the raw measurement uncertainty is investigated, the next step is to establish the VAR model. The optimum VAR order $\mathrm{p}$ has been determined to be as 16 . This value is otained by asserting the minimum values of $\mathrm{AIC}(\mathrm{p})$ and $\mathrm{BIC}(\mathrm{p})$ function. Hence, VAR (16) model for $8298 \times 4$ acceleration data points is resulted in 64x4 VAR parameters and 8282x4 residuals for each damage scenario case. VAR(16) parameters distributions for various duplicated tests at case 1 for channel 4 and 5 are given in Fig 7.a and Fig 7.b, respectively. In order to assess whether there is significant difference between these VAR parameters, they are processed using ANOVA statistical analysis. The $\mathrm{F}$ ratios and $\mathrm{p}$ values for the duplicated test from the channel 1, 2, 3 and 4 have been found as ( $F=3.7487 e-09$, $\mathrm{p}=1.0),(\mathrm{F}=1.2992 \mathrm{e}-08, \mathrm{p}=1.0),(\mathrm{F}=2.0614 \mathrm{e}-09, \mathrm{p}=1.0)$ and $(\mathrm{F}=9.2864 \mathrm{e}-10, \mathrm{p}=1.0)$, respectively. There is not a statistical difference between each repeated measurement ( $>0.05$ ) which may show that any test data that will process in learning algorithms may not effects the classification performance.

The computational results of the VAR parameters and residuals obtained by VAR(16) model for all cases are shown in Fig 8 and 9, respectively. It can be seen that the residuals of the 2nd channel sensor exhibits more random distributions due to it is aligned with the axis of random excitation 

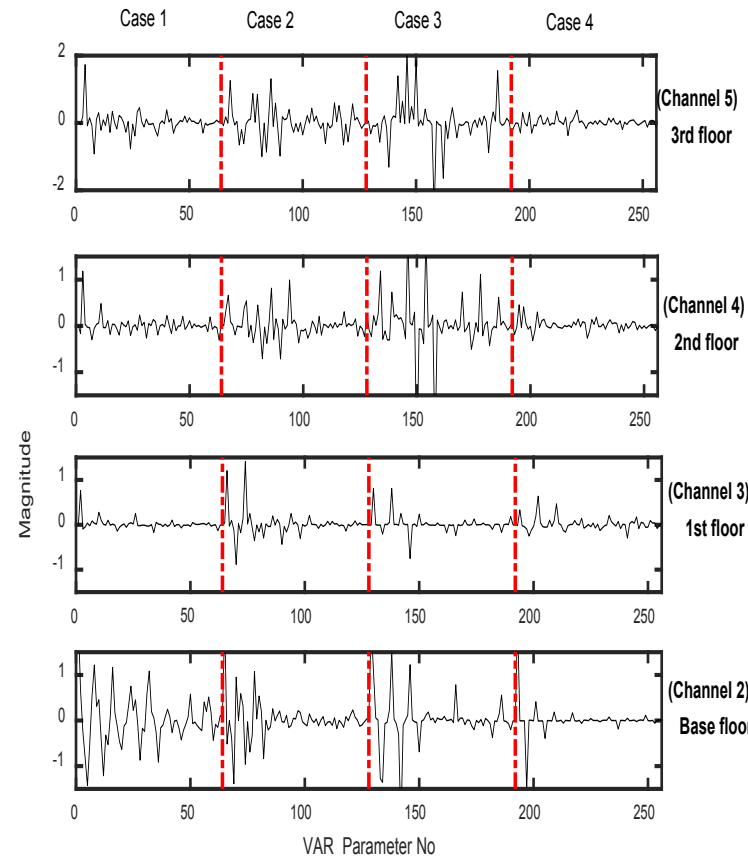

Figure 8. Typical VAR(16) parameters of the 1st dataset
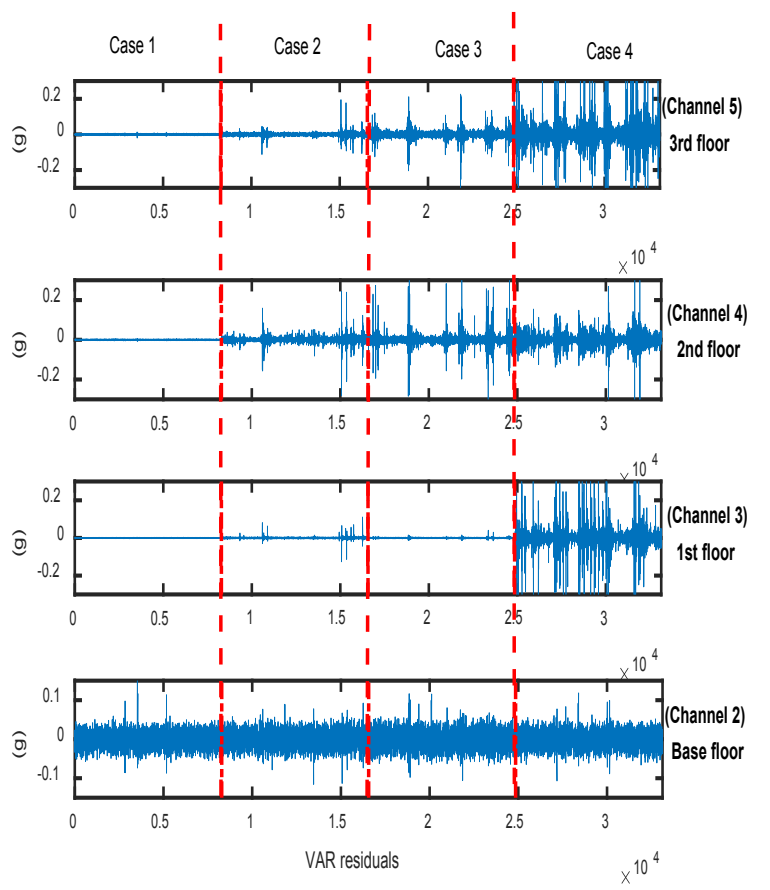

Figure 9. Typical VAR(16) residuals for the 1st dataset

(Fig 9).

Fig 10 represents a typical SVM diagram with the decision boundary for a healthy (Case 1) and damaged case (Case 4). This diagram can be used to improve the shape of decision by modifying the kernel type and its parameters. For instance, the decision boundaries for polynomial $(\mathrm{p}=2)$ and radial type kernel are given in Fig 10.a and 10.b, respectively. a)

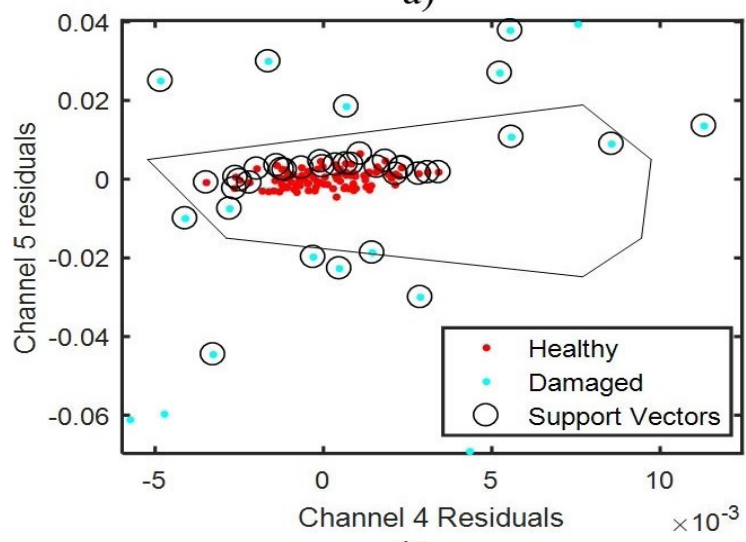

b)

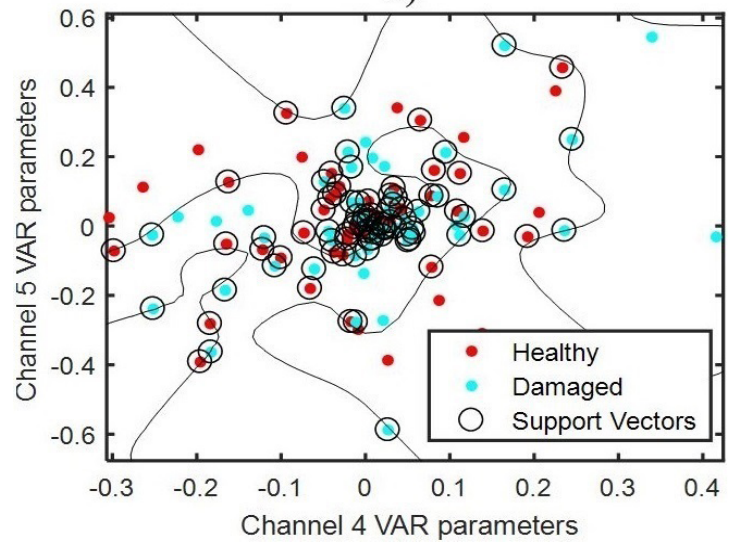

Figure 10. A typical scatter diagram with the decision boundary for VAR(16) model. a) VAR residuals, b) VAR parameters

The damage diagnosis scores are assessed by receiver operating characteristic (ROC) curves. These curves summarizes the performance of the classifiers. They are typically built by plotting the false positive rate (FPR) and true positive rate (TPR) in decision threshold. Once the curve closes the correct positive ratio (TPR) axis the success increases, while it closes false positive ratio (FPR) axis the score decreases. Therefore, a curve with high performance passes through a point at top left side $(0,1)$ of the graph. Each point on the curve complies with a certain threshold and the overall performance of the curve is evaluated in terms of area under curve (AUC). The highest score is obtained when the area under curve (AUC) is equal to 1 , while the worst is 0.5 [25]. As mentioned before, each structural case investigated in this study includes 4 repeated measurements. These datasets are categorized as $50 \%$ for training, $25 \%$ for verifications and the rest of them for testing datasets. The optimal parameters of the kernels are obtained with cross validation.

In first algorithm raw data measurements have used as feature vectors for the SVM classifier. Here, 500x4 feature vectors from each case have been used to establish the SVM model with an optimum radial bases type kernel. One vs. one type approach is used for binary multi classifications. 
Fig 11 represents the ROC curves of SVM technique for raw data observations. It can be seen that AUC values of around 0.5 are obtained, which indicate that the SVM with raw measurements could not discriminate structural states from each other.

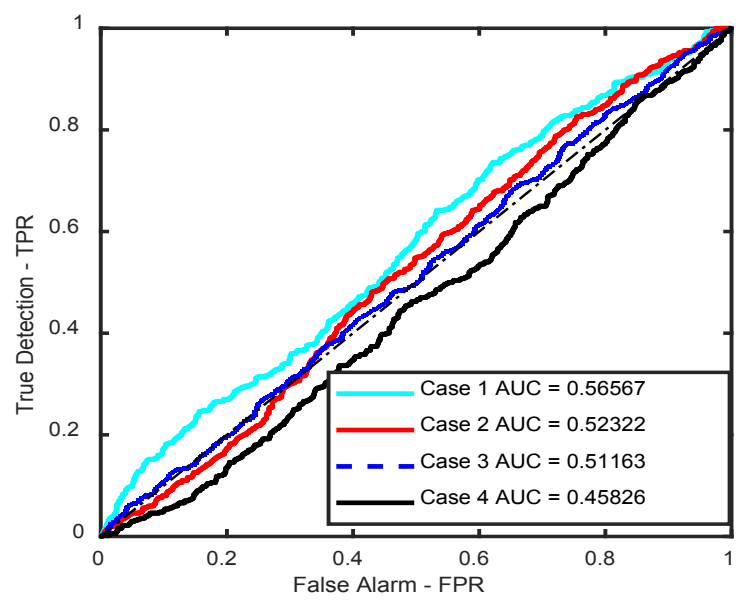

Figure 11. ROC curves of the raw signals processed in SVM model for each case

The performance of each damage diagnosis techniques can further be assessed by confusion matrix plot. The diagonal elements of this matrix show the percentage score for which the estimated case is equal to true case, while off diagonal elements are those that false diagnosed by the technique. The higher values in diagonal elements allows to determine the performance of the diagnosing classifier particularly at individual scenario. Confusion matrix plot obtained with the raw signals processed in SVM model for each case is shown in Fig 12. Overall, SVM scheme gives a low value of $27.8 \%$ correct classifications for the testing datasets.

In the second algorithm, the VAR parameters with a size of $64 \times 4$ observations from each case have been used as feature vectors in SVM. The best results have been obtained

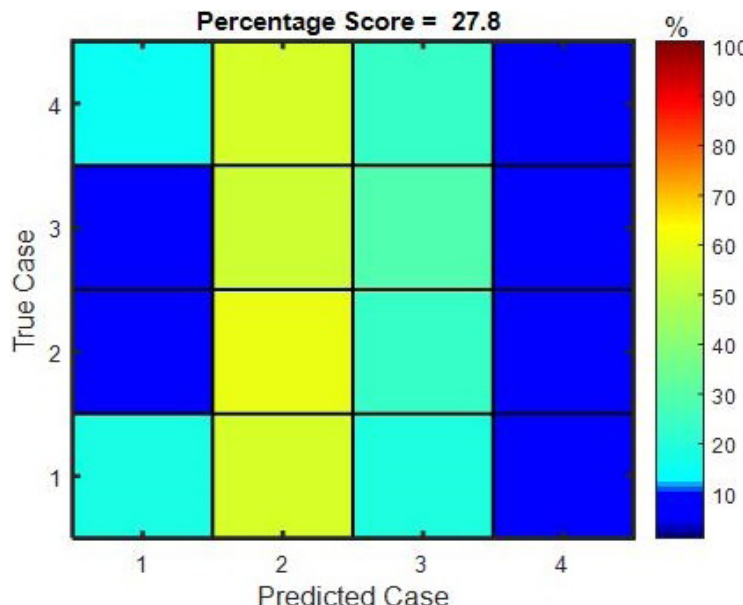

Figure 12. Confusion matrix plot obtained with the raw signals processed in SVM model for each case

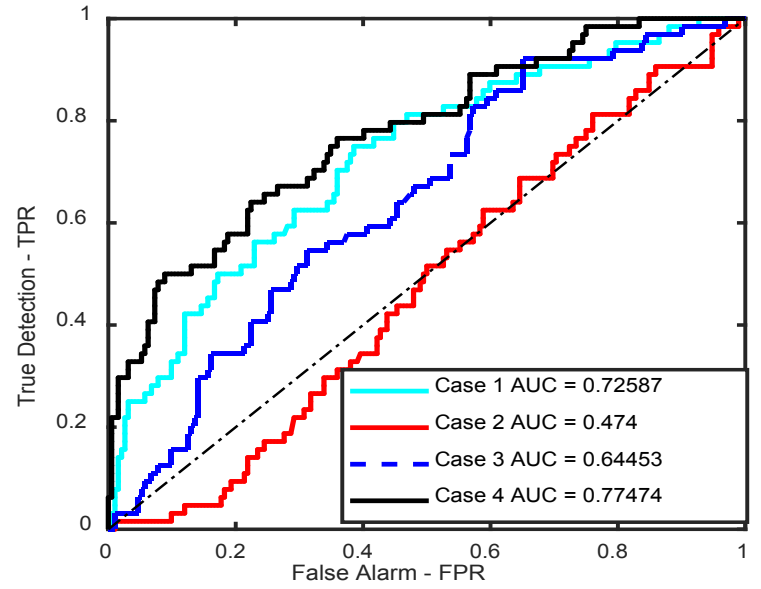

Figure 13. ROC curves of the VAR parameters - SVM model

for RBF type kernel at validation step. ROC curves obtained by VAR SVM technique for each case are represented in Fig 13. AUC values of these curves are $0.7258,0.474,0.6445$ and 0.7747 for case $1,2,3$ and 4 , respectively. It is clear from these results that the classification performance has been improved when the raw observations are processed in VAR model. The maximum correct classification performance is obtained for the case 1 and 4 while the minimum is in case 2 and 3. As the number of the bolt loosening damage increases, higher classifications performance are obtained. This result probably shows that, the more the nonlinear variations in structures, the better the discrimination being reached (Fig 13).

Confusion matrix plot obtained with the VAR parameters features processed in SVM model for each testing case is shown in Fig 14. It is noted that here, the optimal kernel which obtained in validation stage is used during testing. The correct percentage of case 1, 2, 3 and 4 are 81.25, 53.13, 59.38 and 64.06, respectively. As illustrated in Fig 14, the overall performance "VAR Parameters-SVM" model shows

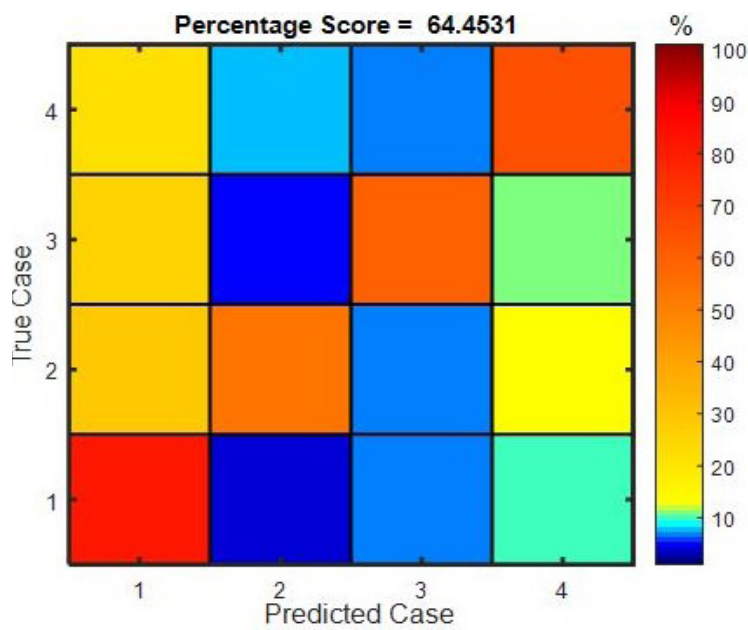

Figure 14. Confusion matrix plot obtained with the VAR parameters SVM damage identification model 


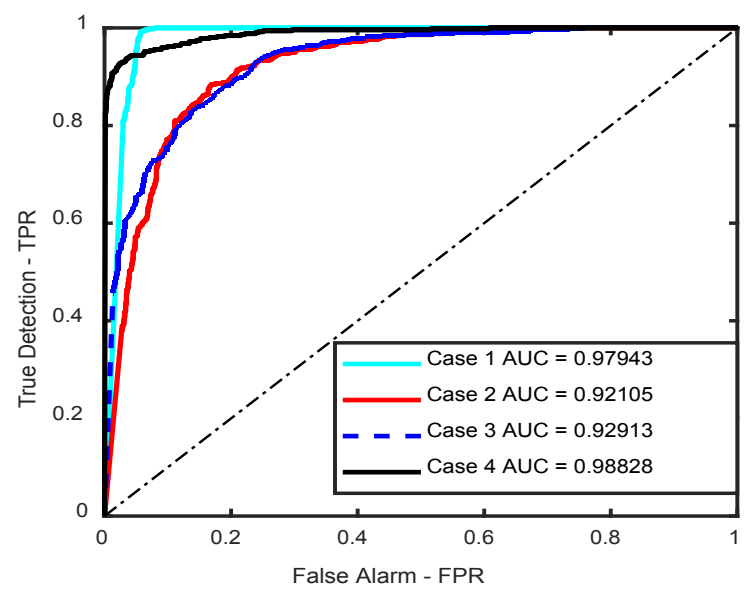

Figure 15. ROC curves of the VAR residuals - SVM diagnosis model

that the algorithm could identify structural damage with a moderate accurate value of $64 \%$. It is also noted that prediction with multi cases is intrinsically more complicated than multiple predictions because of that the algorithm has to learn to construct the separation boundaries or relations of the observation data.

The main objective of the third algorithm is to detect the structural case by evaluating the VAR residuals in SVM model. The residuals with a size of 500x4 observations (1st dataset) from each case have been used as feature vectors for establishing the SVM model. It is observed that a polynomial kernel with an order of 2 gives the best results. The ROC curves obtained from the this approach are given in Fig 15 The highest AUC values of classifying the structural states are found in case 1 and case 4, while the lowest values are in case 2 and 3 .

Finally, the confusion matrix plot obtained in third approach (VAR residuals - SVM) is represented in Fig 16. It is observed that the algorithm diagnoses the correct struc-

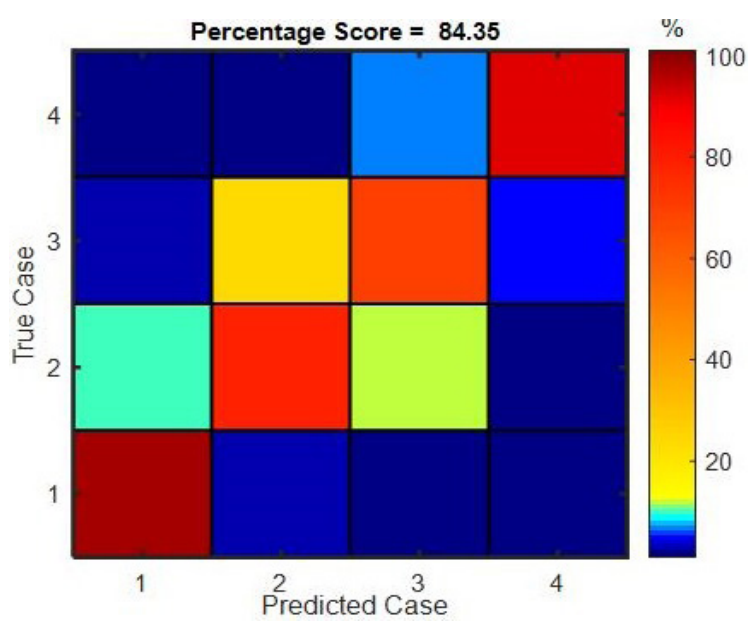

Figure 16. Confusion matrix plot obtained with VAR residuals - SVM damage identification model tural case with a percentage score of 97.4, 78.6, 69.8 and 91.6 for the testing datasets. The false-positive (Type I error) and false-negative (type 2 error) is a common technique that used to determine classification performance. The results of "VAR residuals -SVM" shows a significant performance over both "VAR parameters -SVM" and "raw measurements -SVM" in terms of Type I and Type 2 errors.

Once the algorithm which give the highest accuracy has been determined, the final step is determine its performance for all cases. The ROC curves of the VAR residuals - SVM diagnosis model for all cases are represented in Fig 17. The highest AUC values of classifying the structural case is found in case 1 , while the lowest value is in case 7. It is observed that the algorithm diagnoses the overall correct structural case with a percentage score of 70.25 (Fig 18). Once the performance of the this algorithm for 8 cases compared to 4 cases, the accuracy decreased by $16.15 \%$. This result shows that as the number of case increase, the prediction performance decreases due to separation boundaries as expected.

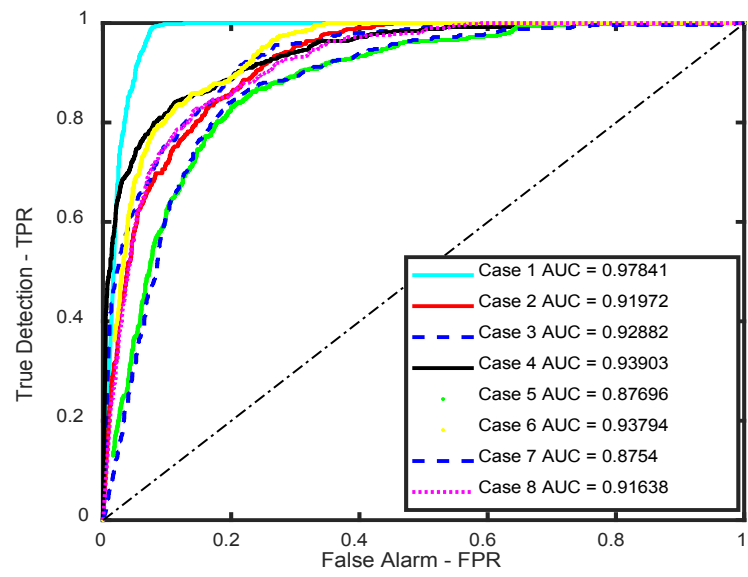

Figure 17. ROC curves of the VAR residuals - SVM diagnosis model for all cases

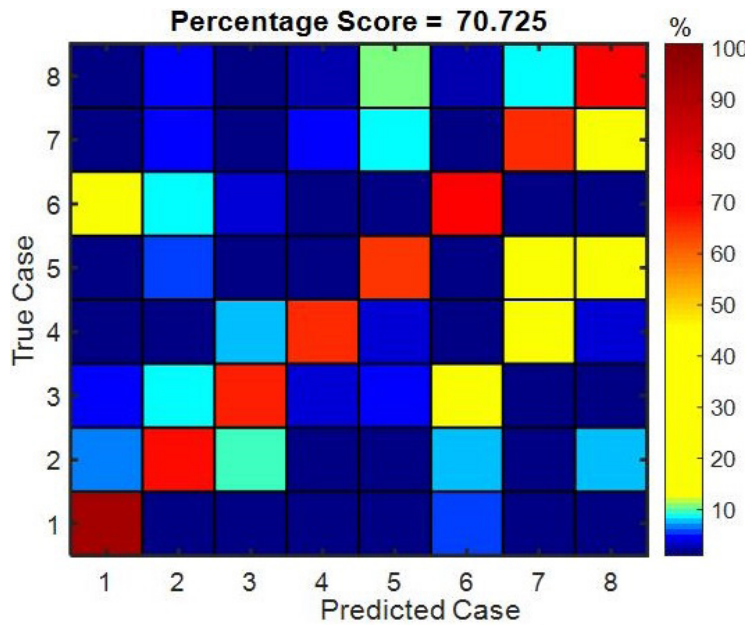

Figure 18. Confusion matrix plot obtained with VAR residuals - SVM damage identification model for all cases 


\section{CONCLUSION}

This study investigates of using time series to diagnose the damages by vector autoregressive - support vector machines . Initially, three floor structure has been manufactured as a test bed. The damage in this structure is simulated by releasing the bolt load which causes a nonlinear effect on the system response. The structure placed on rails is excited by a electrodynamics shaker. The measurements of the four sensors located at different regions have been acquired for various cases. Then, these measurements have been processed in VAR model to generate features of SVM for damage identification. Although improvements have been carried in unsupervised learning structural health monitoring techniques, they are still limited to identify damage sensitive features derived from models of individual sensor. In other words, they are particularly limited to identifying which sensor/sensors is/are associated with the damage $[7,9,10]$. Therefore, we processed the raw measurements in VAR model to improve of damage identification performance. This model captures the interdependencies between multivariate sensor measurements. Once the features such as residuals and parameters are obtained from VAR model, they are divided into learning and testing group for SVM. In order to further summarize the performance of each damage diagnosis methods, the ROC curves for binary classification (healthy, damaged) are given in Fig 19. Note that, while the case 1 is health, the rest cases are the damaged conditions as given in Table 1. The optimum points of ROC curves are determined as $(0.9700$, 0.9920), (0.1146, 0.5156), and (0.05870, 0.9580) for "Raw measurements-SVM", "VAR parameters-SVM" and "VAR parameters-SVM" algorithms, respectively. The first magnitude given in parentheses herein is the "false positive rate" (FPR) or false diagnosis, and the second is the "true positive rate" (TPR) or the correct diagnosis. The most successful diagnosis of damage is achieved when the VAR model residuals are used with SVM. Moreover, it is seen that the binary two case classification scores obtained from each algorithm are higher than multi cases in terms of AUC. This result shows that separating the damaged case from the healthy is easier than separating from another damaged condition. In order to overcome this issue, the stochastic residual errors needs to preprocessed by Markov regime switching Copula model to investigate whether there is a relationship between these errors.

It is expected that the advancements in machine learning procedures will reflect as of increasing their potential uses in SHM applications. One such a machine learning algorithm SVM rises with a number of possible applications. For example considering damage identification problems, an increase in classifier performance may reduce the main-

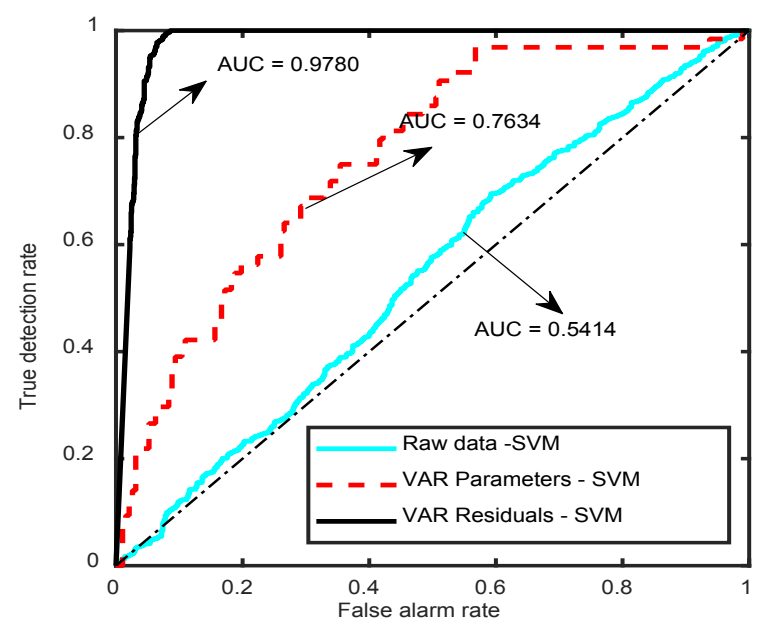

Figure 19. ROC curves of each damage diagnosis model

tenance period requirements for valuable engineering structures. It is noted that the VAR model coupled with Artificial Neural Network (ANN) could also be performed algorithm to detect the damage in engineering structures at environmental and operational conditions. However, the solution $\mathrm{CPU}$ time may lengthen which may not be a practical way particularly for real time SHM applications [20, 26].

The limitations of this study is that the VAR-SVM model has been investigated for a structure in a well controlled laboratory settings. Moreover, this structure is not a scale model of any engineering system and the uncertainties due to humidity and temperature should also be investigated by acquiring large datasets. It is also should be considered whether the created synthetic random excitations reflect the environmental uncertainties. The system this has been designed to validate the algorithms that develop for SHM research. Future struggles should be leaded to deploying of this approach in real physical engineering applications. Additionally, further investigations may focus on the developing a graphical user interface (GUI) for real time damage diagnosing applications. In overall conclusion is that the VAR - SVM has been deployed to diagnose the damages in a well controlled structure. The results indicated that this approach appears to be a promising tool for autonomous SHM implementations.

\section{ACKNOWLEDGEMENT}

The authors would like to thanks Prof. Dr. Hasan Yildiz at Ege University for his valuable suggestions and comments regarding the concepts investigated in this study. This study is partly supported by Ege University, Office of Scientific Research Projects with a designated project ID of 17-ÜYDAUM-001.

\section{REFERENCES}

1. Gopalakrishnan S, Ruzzene M. and Hanagad S. Computational Techniques for Structural Health Monitoring, Springer, London, 
UK, 2011.

2. Pekedis M, Mascarenas D, Turan G, Ercan E., Farrar CR and Yildiz H. Structural health monitoring for bolt loosening via a non-invasive vibro-haptics human-machine cooperative interface. Smart Materials and Structures, 24, 085018, 2015.

3. Pekedis M, Yildiz H. Damage diagnosis of a laminated composite beam and plate via model based structural health monitoring techniques. Journal of the Faculty of Engineering and Architecture of Gazi University, 31(4), 813-831, 2016.

4. Avendaño Valencia L.D, Fassois SD. Damage/fault diagnosis in an operating wind turbine under uncertainty via a vibration response Gaussian mixture random coefficient model based framework Mechanical Systems and Signal Processing, 91, 326-353, 2017.

5. Doebling S, Farrar C., Prime M. and Shevitz D. A review of damage identification methods that examine changes in dynamic properties. Shock Vib. Dig., 30, 91-105, 1998.

6. Farrar C, Doebling S. and Nix D. Vibration based structural damage identification. Philos Trans R Soc A., 356(1778), 131-149, 2001.

7. Farrar CR and Worden K. Structural Health Monitoring a Machine Learning Perspective, Chichester: John Wiley \& Sons Ltd., 2013.

8. Worden K. and Lane AJ. Damage identification using support vector machines. Smart Materials and Structures, 10, 540-547, 2001

9. Bornn L, Farrar CR, Park G, Farinholt K., Sructural health monitoring with autoregressive support vector machines. Journal of Vibration and Acoustics, 131(2), 021004, 2009.

10. Figueiredo E, Park G, Farrar CR, Worden K., Figueiras J. Machine learning algorithms for damage detection under operational and environmental variability. Structural Health Monitoring, 10(6), 559-72, 2011.

11. Sohn H, Farrar CR. Damage diagnosis using time series analysis of vibration signals. Smart Materials and Structures, 10, 446-451, 2001.

12. Sohn H, Robertson AN. and Farrar CR. Holder exponent analysis for discontinuity detection. Structural Engineering and Mechanics An Int'l Journal, 17(3), 409-428, 2004.

13. Allen D, Sohn H, Worden K. and Farrar C. Utilizing the sequential probability ratio test for building joint monitoring. Proc SPIE, 4704, 2002 .
14. Ma J, Perkins S. Online novelty detection on temporal sequences. Proceedings of the 9th ACM SIGKDD International Conference on Knowledge Discovery and Data Mining, Washington DC, 613-618, 2003.

15. Johansen S. Likelihood -based inference in co integration vector auto-regressive models, Oxford University Press Inc, Newyork, USA, 1995.

16. Krolzig H.M. Markov-Switching vector auto regressions, Modelling, Statistical Inference, and Application to Business Cycle Analysis, Springer-Verlag, Berlin, Heidelberrg, Newyork, USA, 1997.

17. Ljung L, System identification -theory for the use, Prentice Hall, Englewood Cliffs, NJ, USA, 1987.

18. Schwarz G. Estimating the dimension of a model. Annals of a statiscs, 6(2), 461-464, 1978.

19. Vapnik VN. The Nature of Statistical Learning Theory, SpringerVerlag, New York, USA, 1998.

20. Bishop CM. Pattern recognition and machine learning, SpringerVerlag, New York NY, USA, 2006,

21. Osuna EE, Freund R. and Girosi F. Support vector machines: training and applications, A,I. Memo No: 1602, C.B, C.L, 144, Massachusetts, 1997.

22. Haykin S. Neural Networks, A comprehensive Foundation, Macmillan, London, UK, 1995.

23. Osabe $\mathrm{K}$ and Kato $\mathrm{T}$. Estimation of standards compliance uncertainty for radiated emission measurement in the PT program, inProc. IEEE Int.Symp. Electromagn. Compat., Long Beach, CA, USA, Aug. 14-19, pp. 994-998, 2011.

24. Carobbi C.F.M, Lalléchère S. and Arnaut L.R. Review of Uncertainty Quantification of Measurement and Computational Modeling in EMC Part I: Measurement Uncertainty," in IEEE Transactions on Electromagnetic Compatibility, 61(6), 1690-1698, 2019.

25. Fawcett T. An introduction to ROC analysis, Pattern Recogn. Lett. 27, 861-874, 2006

26. Raczko E and Zagajewski B. Comparison of support vectormachine, random forest and neural network classifiers for tree species classification on airbornehyperspectral APEX images, European Journal of Remote Sensing, 50(1), 144-154, 2017. 